Assmann, D. - Lektoren, Hörbücher, Events

\title{
Lektoren, Hörbücher, Events - Zur Neufassung des Handbuchs Literaturbetrieb in Deutschland
}

David-Christopher Assmann*

ARNOLD, Heinz Ludwig; BEILEIN, Matthias (Hg.): Literaturbetrieb in Deutschland. 3., völlig veränderte Auflage. Neufassung. Edition Text + Kritik München, 2009. 440 Seiten. ISBN 978-3-88377-996-6.

\section{Literatur, Literaturbetrieb, Literaturwissenschaft}

Als Jens Jessen jüngst zum Auftakt einer Diskussion im Jahrbuch der deutschen Schillergesellschaft fragte, ob denn der gegenwärtige Literaturbetrieb die Literatur nicht ,verderbe' (JESSEN 2007), provozierte er damit Antworten, die sich vor allem im Gestus der Problematisierung der Frage selbst übten: „[D]enn was ist mit ,der Literaturbetrieb' gemeint, und wie soll man so ganz allgemein dessen Einfluß auf die Literatur insgesamt einschätzen können?“ (DREWS 2008: 481). Doch so wenig die Frage, ob und wie literarische Texte und ihre Autorinnen und Autoren unter den gegenwärtigen Betriebsbedingungen ,leiden', sich mit dem Hinweis auf eindeutige Ableitungsverhältnisse zwischen ,Betrieb' und ,Literatur' beantworten lässt, so wenig kann die Literaturwissenschaft auf die Auseinandersetzung mit eben diesen gesellschaftsstrukturellen und institutionellen Voraussetzungen der Produktion, Vermittlung und Rezeption von Literatur verzichten. Literarische Texte sind immer auch in gesellschaftliche, seien es wirtschaftliche, politische oder ganz allgemein soziale Kontexte eingebettet. Und nicht zuletzt deshalb hat sich die Beschäftigung mit dem, was grob unter dem Label ,Literaturbetrieb' firmiert, denn auch mittlerweile als ein

\footnotetext{
* Kollegiat des Deutsch-Italienischen Promotionskollegs der Universitäten Bonn und Florenz. www.germanistik.uni-bonn.de/dipd
} 


$$
\text { Assmann, D. - Lektoren, Hörbücher, Events }
$$

wichtiges und nicht mehr wegzudeckendes Feld (nicht nur) der germanistischen Literaturwissenschaft in Forschung und Lehre etabliert.

\section{Konzentration, Beschleunisung, Marketing}

Die von Heinz Ludwig ARNOLD und Matthias BEILEIN herausgegebene Neufassung des Handbuchs Literaturbetrieb in Deutschland, das 1971 erstmals erschien und 1981 durch eine zweite, völlig veränderte Auflage bereits einmal aktualisiert worden ist, kommt diesen gewachsenen Ansprüchen mit geballter fachlicher Kompetenz nach und bietet der literaturwissenschaftlichen Erforschung der vielfältigen Entstehungsbedingungen von Literatur in der deutschsprachigen Gegenwartsgesellschaft eine mindestens ebenso vielfältige wie informative Grundlage. Neben Bodo Plachtas Überblick über den Literaturbetrieb (PlACHTA 2008) und Stefan Neuhaus Einführung in die Literaturvermittlung (NEUHAUS 2009) trägt damit auch der germanistische Klassiker in Fragen Literaturbetrieb insbesondere den sich durch sozial-, ökonomie- und medienstrukturelle Faktoren seit einigen Jahren abzeichnenden Wandel von Wertungsroutinen in der literarischen Öffentlichkeit durch eine Neuauflage Rechnung. Matthias Beilein bringt diese tiefgreifenden Entwicklungen im Literaturund Kulturbetrieb in seinem Beitrag prägnant auf den Punkt, wenn er festhält, dass

[d]ie Konzentration in Buchhandel und Verlagswesen, die Beschleunigung in der Titelproduktion, der gestiegene Einfluss von Literaturagenten, gesteigerte Rentabilitätserwartungen von Verlags- bzw. Konzernleitung, die Digitalisierung der Kommunikation und des Publizierens, der gestiegene Stellenwert des Marketings und die Veränderungen im Marketing von produktorientiertem zu zielgruppenorientiertem Denken etc. (32)

das Handeln von Produzenten, Vermittlern und Lesern von Literatur in Deutschland maßgeblich verändert haben und dies in Zukunft auch weiter tun werden. Doch wie wirken sich diese Veränderungen, die immer wieder auch Gegenstand heftiger Debatten und Kontroversen (nicht nur in der Literaturkritik) sind, ganz konkret auf die Arbeit im Literaturbetrieb aus? Wie beeinflussen etwa Marktkonzentration, Marketing und Beschleunigung das Handeln der Akteure und Vermittlungsinstanzen? Welche (neuen) Anforderungen werden an Vermittlungsstrategien gestellt? Und lassen sich aus diesen 


$$
\text { Assmann, D. - Lektoren, Hörbücher, Events }
$$

veränderten Ansprüchen Prognosen für die Zukunft erstellen? Kurz: Was bedeutet ,Literaturbetrieb in Deutschland'? Die Herausgeber und mit ihnen die Mitarbeiterinnen und Mitarbeiter des Bandes möchten diesen und ähnlichen Fragen durch ein „Panorama kritischer, teils dezidiert subjektiver Auseinandersetzungen“ (10) mit unterschiedlichsten Aspekten des deutschsprachigen literarischen Lebens begegnen.

\section{Berufsbilder, Märkte, Medien}

Fünf Blöcke strukturieren die insgesamt 30 Beiträge des Handbuchs: Die Leserinnen und Leser erhalten Einblicke in gegenwärtige Tendenzen in den Bereichen „Berufsbilder“, „Vermittler“, „Märkte und Medien“, „Literatur und Öffentlichkeit“" und „Grenzüberschreitungen“. Die auf den ersten Blick recht vage inhaltliche Bestimmung dieser Blöcke wird dabei durch die im Einzelnen präzisen und Interesse weckenden Titel der Beiträge, die jeweils im Untertitel ein systematisierendes Schlagwort aus dem Literaturbetrieb mitführen, aufgefangen: Denn jeder einzelne Beitrag ist schließlich der Ort, an dem sich der je spezifische Einblick in ein bestimmtes Feld des insgesamt immer wieder als ,diffuses Phänomen' etikettierten literarischen Lebens ergibt. Und gerade diese Vielstimmigkeit spiegeln die Beiträge des Handbuchs in ihrer Gesamtheit auch wider.

Der erste Block beschäftigt sich mit Berufsbildern im Literaturbetrieb. Die Spannweite reicht hier von Verlegern über Literaturagenten bis hin zu Buchhändlern. Dabei spielen gerade für die Berufsprofile die angesprochenen Veränderungen im Literaturbetrieb eine wichtige Rolle, agieren doch letztlich alle Akteure des Literaturbetriebs in einem „Raum zwischen Geist und Kommerz“ (15), wie Verleger Thedel Wallmoden in seinem einleitenden Artikel konstatiert. Und auch Karin Fleischanderl weiß aufgrund ihrer Erfahrungen als literarische Übersetzerin, dass ,[d]er höchste Wert [...] Verkäuflichkeit [heißt; DCA]“ (43). Diese Maxime wirkt sich auf die sich meist durch, kontingente Karrierewege' auszeichnenden literaturbetrieblichen Berufsbilder und deren Professionalisierung unmittelbar aus, wie Matthias Beilein in seinem Beitrag für den Beruf des Lektors verdeutlicht. Demnach werde der kulturvermittelnde Aspekt der Lektoratsarbeit, die ,abgesehen von der Leidenschaft für die Literatur und dem fehlerfreien Beherrschen wenigstens der Muttersprache“ (27) 


$$
\text { Assmann, D. - Lektoren, Hörbücher, Events }
$$

keine verbindlichen Schlüsselqualifikationen verlange, in vielen Verlagen nämlich zunehmend ,zugunsten ökonomischer Aspekte in den Hintergrund“ (34) verschoben.

Während es dem ersten Abschnitt noch um Akteure ging, fokussieren die Beiträge des zweiten Blocks auf institutionelle Fragen. Zu den literaturvermittelnden Organisationen und Einrichtungen des Literaturbetriebs zählt das Handbuch unter anderen Autorenverbände, Literaturmuseen oder Sprachakademien. So stellt etwa Rainer Moritz sehr verständlich und trotz nur eines einzigen (wenn auch des wesentlichen!) Literaturhinweises sehr informativ das Selbstverständnis von Literaturhäusern in Deutschland vor, denen es immer auch darum gehe, „das ökonomisch Erfolgreiche nicht in Bausch und Bogen zu verdammen, sondern [...] die Spreu vom Weizen zu trennen“ (127). Moritz' und alle anderen Beiträge dieses Blocks zeichnen sich durch einen hohen Informationsgrad aus, der - zum Beispiel in Jochen Mayers prägnantem Überblick über die Geschichte der Literaturarchive in Deutschland, speziell des wohl einmaligen Marbacher Literaturarchivs - immer auch Platz für Anekdotisches lässt.

Unter dem Titel „Märkte und Medien“ werden Beiträge zusammengefasst, die sich entweder mit Aspekten des deutschen Buchmarkts beschäftigen oder mediale Fragestellungen von Literatur thematisieren. Dazu zählen Beiträge zu Bestsellerlisten, Hörbüchern oder Formen digitaler Literatur. Katrin Blumenkamp stellt kleine und Kleinst-Verlage vor und berichtet dabei nicht zuletzt auch von ihren Erfahrungen mit der Gründung ihres eigenen ,Mini-Verlags'. Nadine van Holt ermöglicht sorgfältig zusammengetragene Einsichten in die Stellung und Bedeutung von Literaturzeitschriften; Hans Sarkowicz gibt einen Überblick über die Geschichte und die aktuelle Bedeutung von Literatur im Radio und weist in diesem Zusammenhang darauf hin, „wie wichtig das Radio weiterhin für Verlage und Autoren ist“ (245). Denn gerade auch das Radio sichere ,die öffentliche Präsenz von Schriftstellern, und [...] erinnert an Werke, die zu Unrecht vergessen sind“ (245). Wolfram Göbel schließlich informiert kompakt über Chancen und Risiken, die sich für Autoren, Texte und Leserinnen aus dem Konzept ,Books on Demand' ergeben.

Die damit bereits angesprochene Frage, welche Spielräume und Möglichkeiten sich durch die Digitalisierung von Literatur ergeben, reichen auch in den Bereich des Verhältnisses von Literaturbetrieb und Öffentlichkeit hinein, das der vierte Block untersucht. Denn hier wird unter anderem „Das Buch und sein Leser im Web 2.0“ 


$$
\text { Assmann, D. - Lektoren, Hörbücher, Events }
$$

diskutiert, wie der Titel des Beitrags von Birte Huizing verspricht. Darüber hinaus geht es diesem Block zum Beispiel um die Bedeutung literarischer Events und den Zusammenhang von Literatur und Justiz. Besonders ins Auge sticht aber der Beitrag von Sabine Buck zum „Kritikerstreit als Betriebsphänomen“, weil er - darin dem Artikel von Tilman Lang und Meike Homann zu Literatur im Fernsehen vergleichbar nicht nur über die wichtigsten Debatten in der Literaturkritik seit 1945 verständlich und komprimiert deskriptiv informiert, sondern diese darüber hinaus auch analytisch näher untersucht. So kann Buck überzeugend skizzieren, dass Literaturdebatten wiederkehrenden Abläufen folgen und die jeweils beteiligten Akteure dabei zumeist typische Argumentationsweisen und Themen verwenden. Dabei sei die literarische Streitkultur seit 1945 nicht zuletzt auch ein Indiz dafür, ,dass das deutsche Feuilleton zunehmend von den Aufmerksamkeitsmechanismen der Massenmedien geprägt wird““ (369). Eher den Charakter eines Exposés hat hingegen Michael Dahnkes Beitrag zu Literaturpreisen, der seine Leser eher mit Fragen denn mit Antworten und Informationen zur Praxis der Preisvergabe in Deutschland zurück lässt.

Der fünfte und letzte Block blickt schließlich über die Grenzen des deutschen Literaturbetriebs hinaus und stellt einerseits die Literaturbetriebe in Österreich und der Schweiz und andererseits den Stellenwert von internationaler Literatur im deutschsprachigen Raum vor. Insbesondere der atemberaubende Parforceritt Doris Mosers durch den österreichischen Literaturbetrieb bietet in diesem Block Möglichkeiten für zahlreiche weitergehendere Überlegungen.

\section{Verderben, Literaturbetrieb, Literatur}

Das Handbuch geht den veränderten Rahmenbedingungen literarischer Kreativität im deutschsprachigen Literaturbetrieb der Gegenwart und den sich daraus für die Literatur ergebenen Chancen und Risiken in insgesamt durchweg informativen und $\mathrm{zu}$ vielerlei Anschlussüberlegungen anregenden Beiträgen nach. Dabei eröffnet nicht zuletzt das sich bereits in den vorherigen Auflagen bewährte Prinzip, neben Literaturwissenschaftlerinnen und Literaturwissenschaftlern auch profilierte Expertinnen und Experten aus der literaturbetrieblichen Praxis zu Wort kommen zu lassen, konkrete und überaus hilfreiche Einblicke in unterschiedlichste Aspekte des 


$$
\text { Assmann, D. - Lektoren, Hörbücher, Events }
$$

gegenwärtigen Literaturbetriebs. Denn es sind nicht zuletzt diese durchaus subjektiven ,Werkstattberichte' und ,Blicke hinter die Kulissen' der Praktiker, die neben den knappen und komprimierten Skizzen historischer Entwicklungen und der pointierten Vermittlung basaler Informationen zum ,Ist-Stand' im Betrieb immer auch wertvolle und gar nicht zu unterschätzende Räume für Anekdotisches, persönliche Erfahrungen und Meinungen eröffnen.

Verdirbt nun der Literaturbetrieb die Literatur? Auf Jens Jessens Frage kann und sollen die Beiträge des Handbuchs keine Antwort geben. Und das nicht, weil selbst ein über 400 Seiten starkes Überblickswerk nicht alle Facetten des Literaturbetriebs abdecken und die Frage damit umfassend beantworten kann - so fehlt etwa ein Artikel zu Poetikvorlesungen, stellen diese doch ohne Frage (auch) ein wichtiges, beliebtes Phänomen im literarischen Leben in Deutschland dar. Der Versuch, die Frage nach dem ,Verderben' der Literatur durch ihren Betrieb zu beantworten, verfinge sich vielmehr in denjenigen Mechanismen von Debatten, Kontroversen und Streitigkeiten in der Literaturkritik, wie sie Sabine Buck in ihrem hervorragenden Beitrag herausarbeitet. Und aus solchen Streitigkeiten sollte die Literaturwissenschaft sich doch besser heraushalten.

Das Handbuch bietet insgesamt einen in dieser Form und in dieser Qualität ohne Frage einmaligen, angesichts der tiefgreifenden Veränderungen im Literaturbetrieb längst überfälligen, nur zu empfehlenden Überblick über aktuelle Entwicklungen des literarischen Lebens in Deutschland. Sein besonderes Merkmal ist dabei die gelungene Kombination aus Überblicksdarstellung einerseits und Detaileinblicken andererseits. Es bietet nicht zuletzt deshalb überaus viele Orientierungshinweise (nicht nur) für Studierende der Germanistik, die sich einen fundierten, sehr verständlichen, hervorragend informierten und dennoch komprimierten Überblick über das verschaffen möchten, was den Literaturbetrieb in Deutschland gegenwärtig charakterisiert. Denn genau das zeigt das Handbuch einmal mehr: dass es den Literaturbetrieb nicht gibt und dennoch (oder gerade deshalb) ist es wichtig, sich mit ihm literaturwissenschaftlich zu beschäftigen. 
Assmann, D. - Lektoren, Hörbücher, Events

\section{Literaturverzeichnis}

DREWS, Jörg. Zum Thema: Verdirbt der Literaturbetrieb die Literatur? In: Jahrbuch der deutschen Schillergesellschaft 52, 2008, 481-491.

JESSEN, Jens. Verdirbt der Literaturbetrieb die Literatur? Vorbemerkung zu einer Diskussion. In: Jahrbuch der deutschen Schillergesellschaft 51, 2007, 11-14.

NeUHAUS, Stefan. Literaturvermittlung. Stuttgart, UVK, 2009.

PLACHTA, Bodo. Literaturbetrieb. Paderborn, Fink, 2008. 